\title{
Pneumonia in exacerbations of COPD: what is the clinical significance?
}

\author{
Samuel P. Trethewey ${ }^{1}{ }^{1}$, John R. Hurst ${ }^{2}$ and Alice M. Turner $\mathbb{1}^{3,4}$ \\ ${ }^{1}$ Oak Tree Surgery, Liskeard, UK. ${ }^{2}$ UCL Respiratory, University College London, London, UK. ${ }^{3}$ Respiratory \\ Medicine, University Hospitals Birmingham NHS Foundation Trust, Birmingham, UK. ${ }^{4}$ Institute of Applied \\ Health Research, University of Birmingham, Birmingham, UK.
}

Correspondence: Samuel P. Trethewey, Oak Tree Surgery, Clemo Road, Liskeard, Cornwall, PL14 3XA, UK. E-mail: s-tretheweydadoctors.org.uk

@ERSpublications

It is vital that clinicians identify radiological consolidation in hospitalised COPD patients, as this confers an increased mortality risk, has important implications for risk stratification and influences management http://bit.ly/2q2vH2J

Cite this article as: Trethewey SP, Hurst JR, Turner AM. Pneumonia in exacerbations of COPD: what is the clinical significance? ERJ Open Res 2020; 6: 00282-2019 [https://doi.org/10.1183/ 23120541.00282-2019].

Does it matter if a patient presenting with an exacerbation of COPD (ECOPD) is found to have consolidation on imaging? In the 2010 European COPD Audit, which included 14111 patients from 384 hospitals in 13 countries with a primary discharge diagnosis of ECOPD, 20\% had concomitant consolidation on admission chest radiography [1]. Crucially, the presence of consolidation was associated with increased 90-day mortality in this cohort (adjusted OR 1.36, 95\% CI 1.2-1.55) [1]. Similar findings were seen in the large 2014 UK National COPD Audit, which found that ECOPD patients with consolidation experienced increased in-hospital mortality $(6.7 \%$ versus $3.6 \%, \mathrm{p}<0.001)$ and increased 90 -day mortality $(15.9 \%$ versus $10.8 \%, \mathrm{p}<0.001)$ compared to patients without consolidation [2].

It is plausible that differences in the pathophysiological mechanisms underlying pneumonic ECOPD ( $\mathrm{pECOPD)}$ and nonpneumonic ECOPD (npECOPD) may partly explain the differences seen in clinical outcomes in these patient groups [3, 4]. GUTIERREZ et al. [3] analysed sputum samples obtained from individuals with pneumonia $(n=15), \operatorname{pECOPD}(n=11)$ and $\operatorname{npECOPD}(n=16)$, and identified differences in the lung microenvironment between groups, comprising different profiles of cytokine generation and specific macrophage activation phenotypes. In a prospective observational cohort study of 249 hospitalised COPD patients, HeurTa et al. [4] found that patients with pECOPD ( $\mathrm{n}=116)$ had significantly higher serum levels of the inflammatory cytokines C-reactive protein, procalcitonin, tumour necrosis factor- $\alpha$ and interleukin-6, compared to patients with npECOPD $(\mathrm{n}=133)$. In addition, a higher rate of Streptococcus pneumoniae ( $43 \%$ versus $10 \%, \mathrm{p}<0.001$ ) and a lower rate of Haemophilus influenzae isolation ( $5 \%$ versus $19 \%, \mathrm{p}=0.04$ ) was observed in patients with $\mathrm{pECOPD}$, compared to patients with npECOPD. Clinical characteristics that were independently associated with PECOPD in this study were: chills, pleuritic pain, sputum purulence and C-reactive protein concentration at day 1 [4]. While these findings need further clarification in larger cohorts, they suggest that hospitalised patients with ECOPD exhibit different clinical profiles based on the presence or absence of comorbid pneumonia.

Current evidence suggests that viruses play a key role in the pathogenesis of ECOPD and $25 \%$ of patients with severe ECOPD appear to experience viral and bacterial co-infection [5]. The natural history of ECOPD is complex and may, in some patients, be characterised by initial viral infection followed by secondary bacterial infection [6]. The progression from acute viral infection to secondary bacterial infection in ECOPD is thought to arise, in part, due to the virally mediated suppression of macrophage

Received: 16 Oct 2019 | Accepted after revision: 20 Nov 2019

Copyright @ERS 2020 This article is open access and distributed under the terms of the Creative Commons Attribution Non-Commercial Licence 4.0. 
phagocytic function and subsequent bacterial outgrowth [7]. However, multiple potential mechanisms may co-exist and further studies are needed to better describe the pathogenesis of viral-bacterial co-infection in ECOPD [8]. Moreover, the influence of, and interaction between, acute pathogenic bacteria and chronically colonised respiratory pathogens requires further clarification in patients with pECOPD. It is also possible that the acute inflammatory state seen in patients with ECOPD confers an increased risk of subsequent pneumonia, adding further complexity to the issue of clinical overlap between ECOPD and pneumonia.

Difficulty in distinguishing pECOPD from npECOPD may arise from a lack of specific biomarkers with sufficient predictive accuracy. For example, previous work has shown that chest radiography has limited sensitivity and positive predictive value for the identification of pulmonary opacities [9]. Computed tomography (CT) has therefore been investigated as an additional imaging modality to better characterise patients with suspected pneumonia [10]. However, there are limited data evaluating the application of CT to patients with ECOPD; in a retrospective observational cohort study of 202 hospitalised patients with an initial diagnosis of ECOPD, 64 patients subsequently underwent CT [11]. In this study, the use of CT altered the primary diagnosis in only two patients (3\%) but resulted in additional diagnoses for 27 patients (42\%), including the identification of pneumonic infiltration in 21 patients (33\%). Interestingly, however, this led to changes in management in only a small number of patients. Most patients were already receiving antibiotics for ECOPD and only one patient discontinued systemic corticosteroids following the identification of consolidation. Crucially, there were no differences in clinical outcomes, including in-hospital mortality, rate of admission to intensive care and length of hospital stay, between patients who underwent CT and those who did not. These preliminary findings suggest that CT may have limited clinical utility in the assessment of patients with ECOPD; however, this requires replication in larger multicentre, prospective cohorts.

In patients with comorbid ECOPD and pneumonia, does it matter which is considered the primary diagnosis? We argue that rather than prioritising one diagnosis over the other, it is more important that the clinical significance of coexistent pneumonia and ECOPD is recognised: these patients experience increased morbidity and mortality $[1,12]$. This has important implications for risk stratification; previous work has shown that traditional pneumonia mortality risk prediction scores perform less well in patients with pECOPD. Specifically, STEER et al. [13] demonstrated that CURB-65 (confusion, urea, respiratory rate, blood pressure, age $\geqslant 65$ years) underestimates mortality risk in this population. The same research group subsequently developed the DECAF score (dyspnoea, eosinopenia, consolidation, acidaemia, fibrillation), which has been shown to better predict mortality risk in patients with pECOPD with an area under the receiver operator characteristic curve of 0.75 (95\% CI $0.71-0.79$ ) for 30-day mortality [14, 15]. Moreover, the DECAF score outperforms CURB-65 in identifying ECOPD patients at low risk of in-hospital mortality, who may be suitable for home treatment [15].

An additional factor to consider is that the management of pneumonia differs from the management of ECOPD. Thus, the question arises of how patients with pECOPD should be managed. A lack of consensus is reflected in international guidelines, which do not offer specific recommendations regarding the management of pECOPD. A key consideration, and source of ongoing debate, is the role of systemic corticosteroids; further, definitive randomised trials are required to determine if patients with $\mathrm{pECOPD}$ should routinely receive systemic corticosteroids [16]. Furthermore, it is unclear which ventilatory strategy is optimal in pECOPD patients with acute respiratory failure. International guidelines do not recommend noninvasive ventilation (NIV) for de novo acute respiratory failure (respiratory failure occurring in the absence of underlying chronic cardiorespiratory disease) in a patient with pneumonia, whereas NIV is recommended for patients with ECOPD [17]. Despite the clear clinical overlap in patients with pECOPD, there are no specific recommendations regarding use of NIV in this population. Recent data from a retrospective observational cohort study of 3971 hospitalised patients with pneumonia requiring ventilation in the USA suggest that initial treatment with NIV, compared to invasive ventilation, is associated with lower in-hospital mortality in patients with comorbid COPD or heart failure (relative risk $0.59,95 \%$ CI $0.47-0.75$ ) but not in patients without these comorbidities (relative risk 0.96, 95\% CI 0.74-1.25) [18]. Furthermore, in this study, the rate of NIV failure was significantly lower in pneumonia patients with comorbid COPD or heart failure $(13.8 \%$ versus $21.3 \%, \mathrm{p}=0.002)$. While these findings appear to support the use of NIV in patients with pneumonia and comorbid COPD or heart failure, it is important to recognise the limitations of this study. Results could have been influenced by unmeasured confounding factors due to the retrospective study design, and the use of the ninth revision of the International Classification of Diseases coding to identify participants could have resulted in misclassification. Further prospective studies are required to clarify the role of NIV in patients with pECOPD and to inform the development of clinical guidelines for this patient group.

Despite the importance of identifying coexistent pneumonia and ECOPD, recent data from a UK-based, retrospective observational cohort study of 621 hospitalised COPD patients suggest that many patients 
with radiographic consolidation do not have a diagnosis of pneumonia recorded on discharge [19]. FINNEY et al. [19] noted that of 235 admissions with confirmed consolidation on chest radiograph, only 101 (43\%) had a diagnosis of pneumonia recorded on discharge, whilst 126 (54\%) had a diagnosis of ECOPD. Only nine patients had both ECOPD and pneumonia listed on their discharge summary. The authors observed clear differences in the frequency of systemic corticosteroid use between patients with a discharge diagnosis of pneumonia, compared to those without ( $56 \%$ versus $98 \%, \mathrm{p}<0.01$ ). Similarly, differences were seen regarding antibiotic prescription: doxycycline was most frequently prescribed in patients diagnosed with ECOPD (47\%) whereas amoxicillin and clarithromycin were most frequently prescribed in patients diagnosed with pneumonia (28\%). These findings suggest that pneumonia is underdiagnosed in hospitalised COPD patients and that diagnosis of comorbid pneumonia is associated with key differences in management.

Finally, evidence suggests that although inhaled corticosteroids (ICS) reduce exacerbation frequency, they increase pneumonia risk in COPD patients [20]. The observed effect appears greatest at higher ICS doses, in older patients and in those with low body mass index, low blood eosinophil counts and severe airflow obstruction [20]. The seemingly paradoxical observation that ICS reduce exacerbation frequency but increase pneumonia risk suggests that ECOPD and pneumonia may have different underlying aetiologies in individual patients. Moreover, the impact of ICS on pneumonia-associated mortality remains unclear; further research is required to evaluate the relative risks versus benefits and to determine whether patients experiencing pECOPD should continue ICS therapy.

In summary, it is vital that clinicians identify radiological consolidation in hospitalised COPD patients, as this confers an increased mortality risk. The DECAF score has greater predictive accuracy for mortality than CURB-65 in patients with pECOPD. Definitive randomised trials are needed to determine if COPD patients with pneumonia should receive systemic corticosteroids and to establish which antibiotics are most effective in these patients. Further research is also required to clarify the role of NIV in patients experiencing pECOPD and the implication of pECOPD for future use of ICS. Recording both diagnoses of ECOPD and pneumonia on discharge is crucial to facilitate meaningful research into this population. To help standardise the assessment and management of patients with comorbid pneumonia and COPD, it may be helpful to agree on a single terminology that encompasses both conditions, such as pECOPD.

Conflict of interest: S.P. Trethewey has nothing to disclose. J.R. Hurst reports support to attend meetings, and payments to himself and to University College London for educational activity and advisory work from pharmaceutical companies that make medicines to treat COPD, outside the submitted work. A.M. Turner reports a research grant for a related systematic review from Grifols Biotherapeutics, research grants for studies in AATD from the Alpha 1 Foundation, personal fees for consultancy work from CSL Behring, and a grant for work in AATD liver disease and expenses to present trial data from Arrowhead Inc., outside the submitted work.

\section{References}

1 Saleh A, López-Campos JL, Hartl S, et al. The effect of incidental consolidation on management and outcomes in COPD exacerbations: data from the European COPD Audit. PLoS One 2015; 10: e0134004.

2 Royal College of Physicians. COPD: who cares when it matters most? - outcomes report 2014. www.rcplondon.ac. $\mathrm{uk} /$ projects/outputs/copd-who-cares-when-it-matters-most-outcomes-report-2014.

3 Gutierrez P, Closa D, Piñer R, et al. Macrophage activation in exacerbated COPD with and without community-acquired pneumonia. Eur Respir J 2010; 36: 285-291.

4 Huerta A, Crisafulli E, Menéndez R, et al. Pneumonic and nonpneumonic exacerbations of COPD: inflammatory response and clinical characteristics. Chest 2013; 144: 1134-1142.

5 Sapey E, Bafadhel M, Bolton CE, et al. Building toolkits for COPD exacerbations: lessons from the past and present. Thorax 2019; 74: 898-905.

6 Welte T, Miravitlles M. Viral, bacterial or both? Regardless, we need to treat infection in COPD. Eur Respir J 2014; 44: 11-13.

7 Wang H, Anthony D, Selemidis S, et al. Resolving viral-induced secondary bacterial infection in COPD: a concise review. Front Immunol 2018; 9: 2345.

8 Hewitt R, Farne H, Ritchie A, et al. The role of viral infections in exacerbations of chronic obstructive pulmonary disease and asthma. Ther Adv Respir Dis 2016; 10: 158-174.

9 Self WH, Courtney DM, McNaughton CD, et al. High discordance of chest x-ray and computed tomography for detection of pulmonary opacities in ED patients: implications for diagnosing pneumonia. Am J Emerg Med 2013; 31: 401-405

10 Claessens YE, Debray MP, Tubach F, et al. Early chest computed tomography scan to assist diagnosis and guide treatment decision for suspected community-acquired pneumonia. Am J Respir Crit Care Med 2015; 192: 974-982.

11 Park HJ, Kim SH, Kim HC, et al. Utility of computed tomography in a differential diagnosis for the patients with an initial diagnosis of chronic obstructive pulmonary disease exacerbation. Tuberc Respir Dis (Seoul) 2019; 82: 234-241.

12 Hurst JR. Consolidation and exacerbation of COPD. Med Sci (Basel) 2018; 6: E44.

13 Steer J, Norman EM, Afolabi OA, et al. Dyspnoea severity and pneumonia as predictors of in-hospital mortality and early readmission in acute exacerbations of COPD. Thorax 2012; 67: 117-121. 
14 Steer J, Gibson J, Bourke SC. The DECAF score: predicting hospital mortality in exacerbations of chronic obstructive pulmonary disease. Thorax 2012; 67: 970-976.

15 Echevarria C, Steer J, Heslop-Marshall K, et al. Validation of the DECAF score to predict hospital mortality in acute exacerbations of COPD. Thorax 2016; 71: 133-140.

16 Niederman MS. Pneumonia complicating COPD: are corticosteroids a help or a hindrance? Chronic Obstr Pulm Dis 2018; 5: 1-4.

17 Rochwerg B, Brochard L, Elliott MW, et al. Official ERS/ATS clinical practice guidelines: noninvasive ventilation for acute respiratory failure. Eur Respir J 2017; 50: 1602426.

18 Stefan MS, Priya A, Pekow PS, et al. The comparative effectiveness of noninvasive and invasive ventilation in patients with pneumonia. J Crit Care 2018; 43: 190-196.

19 Finney LJ, Padmanaban V, Todd S, et al. Validity of the diagnosis of pneumonia in hospitalised patients with COPD. ERJ Open Res 2019; 5: 00031-2019.

20 Agusti A, Fabbri LM, Singh D, et al. Inhaled corticosteroids in COPD: friend or foe? Eur Respir J 2018; 52: 1801219. 